\title{
Temperatura e Alterações no Equilíbrio Ácido-Base de Pacientes Submetidos à Cirurgia Cardíaca com Circulação Extracorpórea, sob Normotermia e Hipotermia *
}

\section{Temperature and Acid-Base Balance in Coronary Bypass Grafting with Cardiopulmonary Bypass, under Hypothermia and Normothermia}

Hugo Leonardo de Moura Luz ${ }^{1}$, José Otávio Costa Auler Junior, TSA ${ }^{2}$

\section{RESUMO}

Luz HLM, Auler Jr JOC - Temperatura e Alterações no Equilíbrio Ácido-Base de Pacientes Submetidos à Cirurgia Cardíaca com Circulação Extracorpórea, sob Normotermia e Hipotermia

Justificativa e Objetivos - A circulação extracorpórea (CEC) associa-se a várias mudanças na fisiologia normal. As múltiplas causas destas alterações interagem e representam um número de rotas potenciais para a disfunção orgânica pós-operatória. O objetivo deste trabalho é investigar as alterações de seus parâmetros indicadores durante a circulação extracorpórea em hipotermia e compará-las àquelas ocorridas em normotermia.

Método - Foram selecionados 30 pacientes adultos, de ambos os sexos, com idades entre 41 e 78 anos, indicados para revascularização cirúrgica do miocárdio, operados com auxílio de CEC, sob normotermia ou hipotermia. Foram avaliados os seguintes parâmetros: concentração de hemoglobina e dos gases sangüíneos, $\mathrm{pH}$, bicarbonato, excesso de bases, hiato aniônico, íon lactato, parâmetros de oxigenação tecidual e os índices de fluxo e de resistência vascular sistêmica.

Resultados - Não houve diferença estatisticamente significativa entre os grupos normotermia e hipotermia, em relação ao pH arterial, ao bicarbonato arterial, às concentrações plasmáticas dos íons sódio e cloreto, ao "anion gap", à pressão parcial de gás carbônico e ao conteúdo arterial de oxigênio. Houve efeito de tempo em todas essas variáveis exceto para as variáveis excesso de bases e "anion gap". O excesso de bases e a concentração de potássio mostraram valores inferiores no grupo hipotérmico. O lactato sérico aumentou nos dois grupos do tempo antes para o após a CEC sendo que o grupo hipotermia apresentou valores mais elevados.

Conclusões - Hipotermia leve parece não modificar substancialmente o equilibrio ácido-base, quando comparado

\footnotetext{
* Recebido do (Received from) Instituto do Coração do Hospital das Clínicas da Faculdade de Medicina da Universidade de São Paulo.

1. Aluno Graduando do $3^{\circ}$ ano da Faculdade de Medicina da Universidade de São Paulo; Bolsista do Programa Institucional de Bolsas de Iniciação Científica do CNPq - Bolsa PIBIC/CNPq - 1999/2000.

2. Professor Titular da Disciplina de Anestesiologia da Faculdade de Medicina da Universidade de São Paulo; Diretor do Serviço de Anestesiologia do InCor - HCFMUSP
}

Apresentado (Submitted) em 23 de abril de 2001

Aceito (Accepted) para publicação em 18 de setembro de 2001

Correspondência para (Mail to):

Dr. José Otávio Costa Auler Junior

Av. Dr. Enéas de Carvalho Aguiar, 255

Bloco $3-8^{\circ}$ Andar - Cerqueira César

05403-900 São Paulo, SP

E-mail: auler@hcnet.usp.br

(c) Sociedade Brasileira de Anestesiologia, 2002 a valores normais de temperatura, durante a CEC. Entretanto, o ion lactato elevou-se significativamente nos pacientes operados sob hipotermia, sugerindo que o transporte de oxigênio para a periferia não foi adequado durante o período de observação proposto. A diminuição da temperatura, embora discreta, parece não ter conferido o grau de proteção celular esperada ao fluxo sangüíneo da CEC.

UNITERMOS - CIRURGIA, Cardíaca: circulação extracorpórea; EQUILÍBRIO ÁCIDO-BASE; HIPOTERMIA; MONITORIZAÇÃO: temperatura

\section{SUMMARY}

Luz HLM, Auler Jr JOC - Temperature and Acid-Base Balance in Coronary Bypass Grafting with Cardiopulmonary Bypass, under Hypothermia and Normothermia

Background and Objectives - Cardiopulmonary bypass $(C P B)$ is related to several changes in normal physiology. The multiple causes of these changes interact and are a potential risk for postoperative organic dysfunction. This study aimed at investigating changes in acid-base and metabolic balance during cardiopulmonary bypass with hypothermia and at comparing them to those observed in patients submitted to normothermal cardiopulmonary bypass.

Methods - Participated in this study 30 adult patients of both genders, aged 41 to 78 years, scheduled for coronary bypass grafting with $C P B$, under normothermia or hypothermia. The following parameters were evaluated: hemoglobin and blood gases concentration, $\mathrm{pH}$, bicarbonate, base excess, anion gap, lactate ion, tissue oxygenation parameters and flow and systemic vascular resistance.

Results - There were no statistically significant differences in arterial $\mathrm{pH}$, arterial bicarbonate, $\mathrm{Na}^{+}$and $\mathrm{Cl}^{-}$plasma concentrations, anion gap, carbon dioxide partial pressure and arterial oxygen content between Normothermia and Hypothermia Groups. A time-effect was observed for all variables except for base excess and anion gap. Base excess and $\mathrm{K}^{+}$concentration were lower in the hypothermia group. Serum lactate increased in both groups when comparing time before $C P B$ to after $C P B$, with higher levels in the hypothermia group.

Conclusions - Mild hypothermia does not seem to substantially change acid-base balance as compared to normal temperature during CPB. Plasma lactate, however, has significantly increased in patients under hypothermia, suggesting inadequate oxygen transport to periphery during the proposed observation period. Low temperature, although mild, does not seem to offer the expected degree of cell protection to CPB blood flow.

KEY WORDS - ACID-BASE BALANCE; HYPOTHERMIA; MONITORING: temperature; SURGERY, Cardiac: cardiopulmonary bypass 


\section{INTRODUÇÃO}

A circulação extracorpórea (CEC) associa-se a várias mudanças na fisiologia normal. As múltiplas causas destas alterações interagem e representam um número de rotas potenciais para a disfunção orgânica pós-operatória ${ }^{1}$. Durante a hipotermia, as variações do $\mathrm{pH}$ têm gênese bioquímica independente das variações da $\mathrm{PaCO}_{2}{ }^{2}$.

Já em 1948 demonstrava-se elevação do $\mathrm{pH}$ com a diminuição da temperatura, independente de quaisquer alterações nas concentrações de bicarbonato, hemoglobina ou proteínas do plasma ${ }^{3}$.

Quando se trata de temperaturas anormais - sendo a hipotermia o melhor exemplo - a variação do $\mathrm{pH}$ pode significar apenas adaptação da fisiologia celular e sangüínea ${ }^{4}$. O complexo imidazol-histidina, além de conferir neutralidade sangüínea com ligeira alcalinidade sobre o pH da água, mantém durante as variações da temperatura, uma faixa de oscilação paralela e semelhante à da água ${ }^{5}$. As vantagens fisiológicas do controle tipo "alfa-stat", para o homem, que é animal homeotérmico, podem ser deduzidas de pesquisas experimentais ${ }^{6,7}$

Acirculação extracorpórea é sistematicamente acompanhada por algum grau de disfunção miocárdica, mesmo quando a hipotermia não é empregada ${ }^{1,8}$. Independente da temperatura atingida ou do protocolo de determinação ácido-base usado ( $\mathrm{pH}$-stat ou pH alfa stat) durante a circulação extracorpórea, ao final do reaquecimento, $\mathrm{pH}$ de 7,4 e $\mathrm{PaCO}_{2}$ superior a $35 \mathrm{mmHg}$ são desejáveis para desconexão segura da bomba. A presença de acidose pode deprimir adicionalmente a contração miocárdica, diminuindo a ação de inotrópicos e aumentando a resistência vascular pulmonar.

Tendo em vista as modificações fisiológicas da circulação extracorpórea nos pacientes em cirurgia cardíaca e as possíveis modificações no equilíbrio ácido-base e metabólico, o objetivo deste trabalho é investigar as alterações de seus parâmetros indicadores durante a circulação extracorpórea em hipotermia e compará-las àquelas ocorridas nos pacientes submetidos à circulação extracorpórea em normotermia.

\section{MÉTODO}

Após ser aprovada pela Comissão de Ética, esta pesquisa foi realizada prospectivamente em 30 pacientes submetidos à cirurgia cardíaca com circulação extracorpórea no Instituto do Coração do Hospital das Clínicas da Faculdade de Medicina da Universidade de São Paulo, no período de outubro de 1999 a junho de 2000 .

Selecionou-se 30 pacientes adultos, de ambos os sexos, com idades entre 41 e 78 anos, indicados para revascularização cirúrgica do miocárdio. Foram excluídos desse estudo pacientes portadores de diabetes mellitus, insuficiência renal ou hepatopatias.

Os pacientes foram divididos em dois grupos de acordo com a temperatura utilizada durante circulação extracorpórea. Grupo Normotermia (N), com pacientes submetidos à cir- culação extracorpórea em normotermia $\left(t=37^{\circ} \mathrm{C}\right)$ e Grupo Hipotermia $(\mathbf{H})$, submetidos à circulação extracorpórea em hipotermia (t entre 32 e $33^{\circ} \mathrm{C}$ ). A temperatura do paciente foi monitorizada com sensor posicionado na nasofaringe, obtida no intraoperatório de forma contínua. O estudo transcorreu durante o período intraoperatório, com avaliação nos seguintes momentos:

\section{M1 - Antes da circulação extracorpórea;}

M2 - Após 15 minutos de circulação extracorpórea;

M3 - Após 30 minutos de circulação extracorpórea;

M4 - Após 60 minutos de circulação extracorpórea;

M5 - Ao final da circulação extracorpórea (tempo padronizado em 15 minutos após a administração de protamina).

Foram colhidas amostras de sangue arterial e venoso dos pacientes, para medida da concentração de hemoglobina $(\mathrm{Hb})$, dos gases sangüíneos e equilíbrio ácido-base: medição das pressões parciais do oxigênio no sangue arterial $\left(\mathrm{PaO}_{2}\right)$ e venoso $\left(\mathrm{PvO}_{2}\right)$, das saturações do oxigênio arterial $\left(\mathrm{SaO}_{2}\right)$ e venoso $\left(\mathrm{SvO}_{2}\right)$, das pressões parciais de gás carbônico arterial $\left(\mathrm{PaCO}_{2}\right)$ e venoso $\left(\mathrm{PvCO}_{2}\right)$; do $\mathrm{pH}$ arterial $(\mathrm{pHa})$ e venoso $(\mathrm{pHv})$, do bicarbonato arterial (Bic art) e venoso (Bic ven), e do excesso de bases arterial (BEa) e venoso (BEv).

Também foi calculado o hiato aniônico ou "anion gap" (VN = 10 a $12 \mathrm{mmol}^{\left.-\mathrm{L}^{-1}\right)}$ para se verificar as possíveis alterações metabólicas do $\mathrm{pH}$. Como a fórmula para o cálculo é $\mathrm{AG}=\mathrm{Na}^{+}$ $-\left(\mathrm{Cl}^{-}+\mathrm{HCO}_{3}^{+}\right)$, foram dosados no plasma o íon sódio (fotometria de chama, $\mathrm{VN}=133$ a $145 \mathrm{mmol} . \mathrm{L}^{-1}$ ) e o cloreto (método calorimétrico, $\mathrm{VN}=98$ a $\left.119 \mathrm{mmol} \cdot \mathrm{L}^{-1}\right)$.

$\mathrm{O}$ íon lactato foi determinado apenas no sangue arterial nos momentos pré-CEC e pós-CEC pelo método enzimático ultravioleta.

Os parâmetros de oxigenação tecidual e os índices de fluxo e de resistência vascular sistêmica foram calculados para os três períodos de CEC a partir de fórmulas apropriadas para a obtenção dos mesmos:

- conteúdo de oxigênio

$\left(\mathrm{CaO}_{2}, \mathrm{VN}=17\right.$ a $20 \mathrm{ml} / \mathrm{dl}$ e $\mathrm{CvO}_{2}, \mathrm{VN}=12$ a $\left.15 \mathrm{ml} / \mathrm{dl}\right)$, $\mathrm{CaO}_{2}=\left(\mathrm{Hb} \cdot \mathrm{SaO}_{2} \cdot 1,34 / 100\right)+\left(\mathrm{PaO}_{2} \cdot 0,0031\right) ;$

- diferença arteriovenosa de oxigênio $\left(\mathrm{DavO}_{2}, \mathrm{VN}=4\right.$ a 5 $\mathrm{ml} / \mathrm{dl}), \mathrm{DavO}_{2}=\mathrm{CaO}_{2}-\mathrm{CvO}_{2}$;

- extração de oxigênio $\left(\mathrm{EO}_{2}, \mathrm{VN}=24\right.$ a $\left.28 \%\right)$, $\mathrm{EO}_{2}=\left(\mathrm{CaO}_{2}-\mathrm{CvO}_{2}\right) / \mathrm{CaO}_{2}$;

- índice de transporte de oxigênio $\left(\mathrm{iTO}_{2}, \mathrm{VN}=550\right.$ a 650 $\left.\mathrm{ml} / \mathrm{min} \cdot \mathrm{m}^{2}\right), \mathrm{iTO}_{2}=\left(\mathrm{CaO}_{2}\right.$. Fluxo $) /($ SupCorp. 100$)$;

- índice de consumo de oxigênio ( $\mathrm{iVO}_{2}, \mathrm{VN}=115$ a 165 $\left.\mathrm{ml} \cdot \mathrm{min}^{-1} \cdot \mathrm{m}^{2}\right), \mathrm{iVO}_{2}=\left(\operatorname{DavO}_{2}\right.$. Fluxo $) /($ SupCorp. 100$)$;

- índice de fluxo, iFluxo=Fluxo/SupCorp;

- índice de resistência vascular sistêmica, iRVS=(PAM.80.1000)/(Fluxo/SupCorp).

O tratamento estatístico dos dados consistiu na análise dos perfis médios de cada grupo (normotermia e hipotermia) 
considerado ${ }^{11}$. A variável-resposta adotada foi o desvio em relação à medida pré-CEC, ou seja, a resposta no tempo $x$ (minutos) é a diferença entre o observado no tempoxe a medida pré-CEC.

Mediante essa análise, as observações pós-CEC foram desconsideradas, uma vez que as mesmas ocorreram em tempos distintos de paciente para paciente. O modelo estatístico utilizado contemplou possíveis correlações entre observações realizadas num mesmo indivíduo; para tal, utilizou-se uma estrutura de correlação não estruturada ${ }^{9}$. Foram realizados testes para algumas hipóteses de interesse como, por exemplo, a de igualdade de comportamento entre grupos nos tempos estudados (teste de coincidência de perfis médios) ${ }^{10}$. O nível de significância utilizado foi de $5 \%$.

\section{RESULTADOS}

Na tabela I, os grupos normotermia $(\mathrm{N})$, com total de 14 pacientes, e hipotermia $(H)$, com 16, estão expostos com os dados referentes a idade, peso, altura e superfície corpórea, não havendo diferença estatística entre os grupos.

Tabela I - Dados Demográficos (Média \pm DP)

\begin{tabular}{lcc}
\hline & Grupo N & Grupo H \\
\hline Idade (anos) & $60,6 \pm 9,2$ & $62,5 \pm 10,3$ \\
Peso $(\mathrm{kg})$ & $77,6 \pm 6,8$ & $75,9 \pm 14,8$ \\
Altura $(\mathrm{m})$ & $1,69 \pm 0,05$ & $1,68 \pm 0,06$ \\
Superfície corpórea $\left(\mathrm{m}^{2}\right)$ & $1,87 \pm 0,11$ & $1,86 \pm 0,21$ \\
\hline
\end{tabular}

$\mathrm{N}$ - Normotermia; $\mathrm{H}$ - Hipotermia

Os valores médios do tempo de circulação extracorpórea e da temperatura mínima durante circulação extracorpórea encontram-se na tabela II. A duração da CEC foi maior no grupo $\mathrm{H}$.
Tabela II - Parâmetros da Circulação Extracorpórea (Média $\pm \mathrm{DP})$

\begin{tabular}{lcc}
\hline & Grupo N & Grupo H \\
\hline Duração da CEC (min) & $87 \pm 19$ & $105 \pm 25$ \\
Temperatura mínima durante a CEC $\left({ }^{\circ} \mathrm{C}\right)$ & $35,2 \pm 1,2$ & $31,9 \pm 1,6$ \\
\hline
\end{tabular}

$\mathrm{N}$ - Normotermia; $\mathrm{H}$ - Hipotermia

A pressão arterial média, o fluxo arterial e a resistência vascular sistêmica foram avaliados durante os três tempos pré-determinados (15, 30 e 60 minutos) de circulação extracorpórea nos dois grupos estudados e os valores médios estão na tabela III. A pressão arterial média e o fluxo arterial serviram para o cálculo de outras variáveis e não foram analisados estatisticamente. Mesmo sem análise estatística, os valores obtidos de resistência vascular periférica nesse trabaIho mostraram-se mais elevados durante a CEC no grupo hipotermia.

Tabela III - Parâmetros Fisiológicos Durante a CEC CEC-15 min CEC-30 min CEC-60 min

Pressão arterial média $(\mathrm{mmHg})$

\begin{tabular}{llll} 
Grupo N & $61,2 \pm 1,3$ & $56,6 \pm 1,3$ & $59,9 \pm 9,7$ \\
Grupo H & $61,2 \pm 9,3$ & $56,3 \pm 9,3$ & $60,0 \pm 9,7$ \\
\hline
\end{tabular}

Índice de fluxo arterial na circulação extracorpórea (L. $\left.\mathrm{min}^{-1} \cdot \mathrm{m}^{-2}\right)$

$\begin{array}{llll}\text { Grupo N } & 2575,5 \pm 523 & 2708,2 \pm 471 & 2648,9 \pm 556 \\ \text { Grupo H } & 2497,8 \pm 350 & 2533,9 \pm 380 & 2414,6 \pm 332\end{array}$

Índice de resistência vascular sistêmica (dyn $\left.\mathrm{seg}^{\mathrm{cm}} \mathrm{cm}^{-5}\right)$

Grupo N 2011,1 $\pm 399 \quad 1868,8 \pm 586 \quad 2134,8 \pm 719$

Grupo H $2128,4 \pm 378 \quad 1957,9 \pm 454 \quad 2169,7 \pm 533$

Os dados comparativos das pressões parciais de $\mathrm{O}_{2}, \mathrm{CO}_{2}$, e os parâmetros de oxigenação tecidual estão apresentados nas tabelas IV, V e VI.

Tabela IV - Parâmetros de Oxigenação e Ventilação (Média \ DP)

\begin{tabular}{|c|c|c|c|c|c|}
\hline & Pré-CEC & CEC- 15 min & CEC - $30 \mathrm{~min}$ & CEC - 60 min & Pós-CEC \\
\hline \multicolumn{6}{|c|}{ Pressão parcial de $\mathrm{O}_{2}$ no sangue arterial $(\mathrm{mmHg})$} \\
\hline Grupo N & $207,9 \pm 82,3$ & $200,3 \pm 72,4$ & $168,8 \pm 66,2$ & $187,3 \pm 60,4$ & $129,3 \pm 43,8$ \\
\hline \multicolumn{6}{|c|}{ Pressão parcial de $\mathrm{O}_{2}$ no sangue venoso $(\mathrm{mmHg})$} \\
\hline Grupo N & $43,3 \pm 5,8$ & $40,9 \pm 6,4$ & $42,0 \pm 5,6$ & $40,8 \pm 5,3$ & $46,4 \pm 7,2$ \\
\hline \multicolumn{6}{|c|}{ Pressão parcial de $\mathrm{CO}_{2}$ no sangue arterial $(\mathrm{mmHg})$} \\
\hline Grupo N & $31,5 \pm 3,7$ & $37,9 \pm 3,3$ & $40,0 \pm 4,7^{*}$ & $39,6 \pm 5,1$ & $36,0 \pm 3,8$ \\
\hline Grupo H & $35,7 \pm 6,0$ & $39,9 \pm 5,6$ & $41,6 \pm 6,1^{*}$ & $38,8 \pm 6,0$ & $38,1 \pm 3,1$ \\
\hline \multicolumn{6}{|c|}{ Pressão parcial de $\mathrm{CO}_{2}$ no sangue venoso $(\mathrm{mmHg})$} \\
\hline Grupo N & $40,8 \pm 3,4$ & $41,5 \pm 2,6$ & $44,3 \pm 3,6$ & $44,9 \pm 4,0$ & $43,7 \pm 2,7$ \\
\hline Grupo H & $42,4 \pm 5,1$ & $42,9 \pm 6,7$ & $44,7 \pm 7,7$ & $43,5 \pm 5,8$ & $42,5 \pm 4,2$ \\
\hline
\end{tabular}

* $p<0,05$ para variação ao longo do tempo 


\begin{tabular}{|c|c|c|c|c|c|}
\hline & Pré-CEC & CEC - 15 min & CEC - 30 min & CEC - $60 \mathrm{~min}$ & Pós-CEC \\
\hline \multicolumn{6}{|c|}{ Conteúdo arterial de $\mathrm{O}_{2}(\mathrm{ml} / \mathrm{dl})$} \\
\hline Grupo N & $18,10 \pm 1,53$ & $13,14 \pm 2,61$ & $12,92 \pm 1,75$ & $13,58 \pm 1,69^{*}$ & $15,83 \pm 1,25$ \\
\hline Grupo H & $19,43 \pm 2,80$ & $12,75 \pm 1,28$ & $12,91 \pm 1,08$ & $13,48 \pm 1,40^{*}$ & $14,24 \pm 1,39$ \\
\hline \multicolumn{6}{|c|}{ Conteúdo venoso de $\mathrm{O}_{2}(\mathrm{ml} / \mathrm{dl})$} \\
\hline Grupo N & $13,83 \pm 1,80$ & $9,86 \pm 2,17$ & $9,49 \pm 1,80$ & $9,76 \pm 1,91$ & $10,89 \pm 1,11$ \\
\hline Grupo H & $15,70 \pm 2,92$ & $9,80 \pm 1,61$ & $10,16 \pm 1,09$ & $10,05 \pm 1,57$ & $11,76 \pm 1,34$ \\
\hline \multicolumn{6}{|c|}{ Diferença arteriovenosa de $\mathrm{O}_{2}(\mathrm{ml} / \mathrm{dl})$} \\
\hline Grupo N & $4,27 \pm 1,18$ & $3,56 \pm 1,02$ & $3,43 \pm 0,88$ & $3,82 \pm 0,75$ & $2,93 \pm 0,92$ \\
\hline Grupo H & $3,73 \pm 1,02$ & $2,95 \pm 0,72$ & $2,75 \pm 0,64$ & $3,43 \pm 0,65$ & $2,48 \pm 0,76$ \\
\hline
\end{tabular}

${ }^{*} p<0,05$ para variação ao longo do tempo

Tabela VI - Parâmetros de Oxigenação Tecidual durante a CEC (Média \pm DP)

\begin{tabular}{|c|c|c|c|}
\hline & CEC - 15 min & CEC - $30 \mathrm{~min}$ & CEC - $60 \mathrm{~min}$ \\
\hline \multicolumn{4}{|c|}{ Índice de resistência vascular sistêmica $\left(\mathrm{din} / \mathrm{s} / \mathrm{cm}^{5}\right)$} \\
\hline Grupo N & $2011,1 \pm 399,0$ & $1868,8 \pm 585,7$ & $2134,8 \pm 718,6$ \\
\hline Grupo H & $2128,4 \pm 377,7$ & $1958,0 \pm 454,5$ & $2169,7 \pm 533,3$ \\
\hline \multicolumn{4}{|c|}{ Índice de transporte de $\mathrm{O}_{2}\left(\mathrm{ml} / \mathrm{min} / \mathrm{m}^{2}\right)$} \\
\hline Grupo N & $323,32 \pm 107,95$ & $333,62 \pm 90,60$ & $344,18 \pm 103,32$ \\
\hline Grupo H & $297,43 \pm 48,51$ & $306,29 \pm 55,72$ & $303,60 \pm 48,39$ \\
\hline \multicolumn{4}{|c|}{ Índice de consumo de $\mathrm{O}_{2}\left(\mathrm{ml} / \mathrm{min} / \mathrm{m}^{2}\right)$} \\
\hline Grupo N & $92,00 \pm 31,01$ & $91,92 \pm 26,28$ & $99,67 \pm 22,86$ \\
\hline Grupo H & $75,19 \pm 28,84$ & $69,04 \pm 17,36$ & $81,84 \pm 14,58$ \\
\hline \multicolumn{4}{|c|}{ Extração de $\mathrm{O}_{2}(\%)$} \\
\hline Grupo N & $27,35 \pm 6,62$ & $26,87 \pm 7,50$ & $28,59 \pm 6,61$ \\
\hline Grupo H & $23,51 \pm 6,76$ & $21,31 \pm 4,94$ & $25,59 \pm 5,69$ \\
\hline
\end{tabular}

As variáveis $\mathrm{PaO}_{2}, \mathrm{PvO}_{2}, \mathrm{PvCO}_{2}, \mathrm{CvO}_{2}, \mathrm{DavO}_{2}, \mathrm{iTO}_{2}$ e EO não foram analisadas estatisticamente e serviram apenas para o cálculo de outros dados.

A pressão parcial de $\mathrm{CO}_{2}$ arterial tem seus perfis médios representados na figura 1. As curvas obtidas mostraram paralelismo, além de coincidência entre elas. A média geral dos desvios nos dois grupos aumentou no intervalo entre 15 e 30 minutos de CEC, sem alterações significativas no período seguinte.

O conteúdo arterial de $\mathrm{O}_{2}$ oscilou de modo semelhante nos dois grupos, e as curvas foram coincidentes, mostrando que não houve diferença estatisticamente significativa nos valores dessa variável entre os dois grupos, com o grupo hipotermia apresentando valores mais baixos. Entre 30 e 60 minutos ocorreu elevação do conteúdo arterial de oxigênio em ambos os grupos.

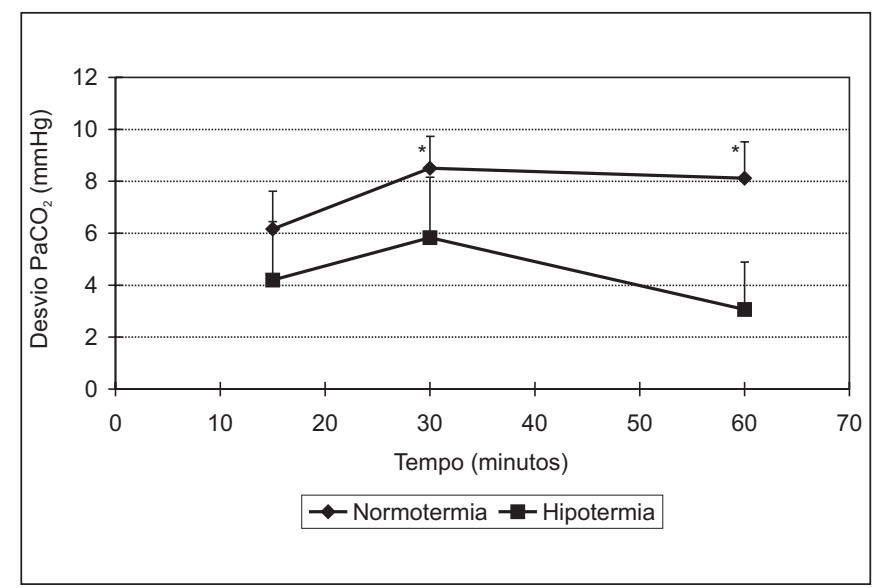

Figura 1 - Perfis Médios para a Variável $\mathrm{PaCO}_{2}$ ${ }^{*} p<0,05$ 
Tabela VII - Indicadores do Equilíbrio Ácido-Base (Média \pm DP)

\begin{tabular}{|c|c|c|c|c|c|}
\hline & Pré-CEC & CEC - 15 min & CEC - 30 min & CEC - 60 min & Pós-CEC \\
\hline \multicolumn{6}{|c|}{$\mathrm{PH}$ arterial } \\
\hline Grupo N & $7,45 \pm 0,068$ & $7,37 \pm 0,068$ & $7,36 \pm 0,077^{*}$ & $7,37 \pm 0,071$ & $7,35 \pm 0,077$ \\
\hline Grupo H & $7,46 \pm 0,039$ & $7,39 \pm 0,031$ & $7,37 \pm 0,042^{*}$ & $7,38 \pm 0,049$ & $7,38 \pm 0,035$ \\
\hline \multicolumn{6}{|c|}{ Excesso de bases arterial $\left(\mathrm{mmol} . \mathrm{L}^{-1}\right)$} \\
\hline Grupo N & $-0,29 \pm 1,79$ & $-1,76 \pm 2,12$ & $-1,42 \pm 2,04$ & $-1,25 \pm 1,78$ & $-3,03 \pm 2,06^{* *}$ \\
\hline \multicolumn{6}{|c|}{ Concentração de $\mathrm{Na}^{+}\left(\mathrm{mmol} . \mathrm{L}^{-1}\right)$} \\
\hline Grupo N & $137,86 \pm 1,41$ & $129,36 \pm 3,20$ & $130,00 \pm 2,25$ & $131,00 \pm 2,18^{*}$ & $133,21 \pm 3,84$ \\
\hline Grupo H & $137,62 \pm 2,09$ & $130,81 \pm 3,21$ & $130,69 \pm 2,12$ & $131,75 \pm 1,73^{*}$ & $131,00 \pm 2,18$ \\
\hline \multicolumn{6}{|c|}{ Concentração de $\mathrm{K}^{+}\left(\mathrm{mmol} . \mathrm{L}^{-1}\right)$} \\
\hline Grupo N & $3,74 \pm 0,43$ & $4,28 \pm 0,39^{*}$ & $4,36 \pm 0,47^{*}$ & $4,66 \pm 0,48^{*}$ & $3,77 \pm 0,41^{* *}$ \\
\hline Grupo H & $3,90 \pm 0,30$ & $3,82 \pm 0,50^{*}$ & $4,16 \pm 0,67^{*}$ & $4,50 \pm 0,60^{*}$ & $3,76 \pm 0,36^{* *}$ \\
\hline \multicolumn{6}{|c|}{ Concentração de $\mathrm{Cl}^{-}\left(\mathrm{mmol} . \mathrm{L}^{-1}\right)$} \\
\hline Grupo N & $101,43 \pm 3,91$ & $97,93 \pm 3,07$ & $97,57 \pm 2,82$ & $98,93 \pm 2,30^{*}$ & $100,07 \pm 2,61$ \\
\hline Grupo H & $100,56 \pm 2,13$ & $98,87 \pm 2,75$ & $98,62 \pm 2,36$ & $99,50 \pm 2,78^{*}$ & $100,69 \pm 2,50$ \\
\hline \multicolumn{6}{|c|}{ Concentração de $\mathrm{HCO}_{3}^{-}$arterial $\left(\mathrm{mmol} . \mathrm{L}^{-1}\right)$} \\
\hline Grupo N & $23,00 \pm 1,75$ & $21,57 \pm 1,88$ & $22,01 \pm 2,01^{*}$ & $22,31 \pm 2,09$ & $19,94 \pm 2,09$ \\
\hline Grupo H & $23,30 \pm 2,16$ & $22,77 \pm 1,38$ & $23,24 \pm 1,54^{*}$ & $23,01 \pm 2,01$ & $21,44 \pm 2,85$ \\
\hline \multicolumn{6}{|c|}{ Anion Gap (mmol. $\left.\mathrm{L}^{-1}\right)$} \\
\hline Grupo N & $13,43 \pm 2,85$ & $9,86 \pm 3,05$ & $10,41 \pm 2,83$ & $9,76 \pm 3,07$ & $13,20 \pm 5,54$ \\
\hline Grupo H & $13,76 \pm 3,51$ & $9,16 \pm 3,06$ & $8,82 \pm 2,68$ & $9,24 \pm 2,85$ & $13,43 \pm 4,22$ \\
\hline \multicolumn{6}{|c|}{ Íon lactato $\left(\mathrm{mmol} . \mathrm{L}^{-1}\right)$} \\
\hline Grupo N & $15,27 \pm 6,04$ & & & & $25,43 \pm 11,16^{* *}$ \\
\hline Grupo H & $16,72 \pm 8,18$ & & & & $44,84 \pm 29,15^{\text {** }}$ \\
\hline
\end{tabular}

* $p<0,05$ para variação ao longo do tempo

${ }^{* *} p<0,05$ para diferença entre os grupos

A variável consumo de oxigênio, apesar de não submetida à análise estatística, mostrou valores em média mais elevados no grupo normotermia nos momentos estudados durante a CEC.

$\mathrm{O}$ pH sangüíneo e o excesso de bases foram analisados no sangue arterial e venoso e os dados obtidos estão na tabela VII. Foram obtidos parâmetros referentes às concentrações de sódio ionizado, íon cloreto e bicarbonato para o cálculo do hiato de aniônico (ou "anion gap" ou AG). As concentrações de lactato medidas nos períodos pré-CEC e pós-CEC também encontram-se na tabela VII.

A concentração do íon sódio no sangue arterial variou de modo semelhante entre os dois grupos, com as curvas obtidas coincidentes estatisticamente. Em relação à variação temporal, apenas ocorreu aumento significativo nas médias gerais dos desvios no intervalo entre 30 e 60 minutos de CEC.

Em relação ao íon potássio, sua concentração sérica variou de modo semelhante; no entanto, os valores médios foram estatisticamente diferentes entre os dois grupos, com o grupo normotermia apresentando valores mais elevados duran- te a CEC. Houve aumento da média geral ao longo do tempo, demonstrando o efeito temporal.

Para a concentração do ânion cloreto também foram obtidas curvas paralelas e coincidentes, sendo que a alteração temporal foi estatisticamente significativa apenas entre 30 e 60 minutos de CEC.

$\mathrm{O} \mathrm{pH}$ arterial variou de modo semelhante entre os dois grupos e as curvas dos perfis médios ao longo do tempo (Figura 2) foram coincidentes a partir da análise estatística. Entre 15 e 30 minutos de CEC houve diminuição dos valores médios gerais e, apesar de não ter sido detectada variação estatisticamente significativa entre 30 e 60 minutos, a média geral neste último tempo foi igual à do tempo 15 minutos.

Com relação ao excesso de bases no sangue arterial, as curvas de desvio dos grupos foram paralelas não coincidentes, além de não ter sido detectada alteração ao longo do tempo (Figura 3).

O bicarbonato arterial apresentou variação semelhante entre os tempos nos dois grupos. Também não houve diferença estatisticamente significativa entre os valores médios de cada curva, isto é, elas foram coincidentes. Entre 15 e $30 \mathrm{mi}-$ nutos de CEC ocorreu elevação da média geral. No entanto, 


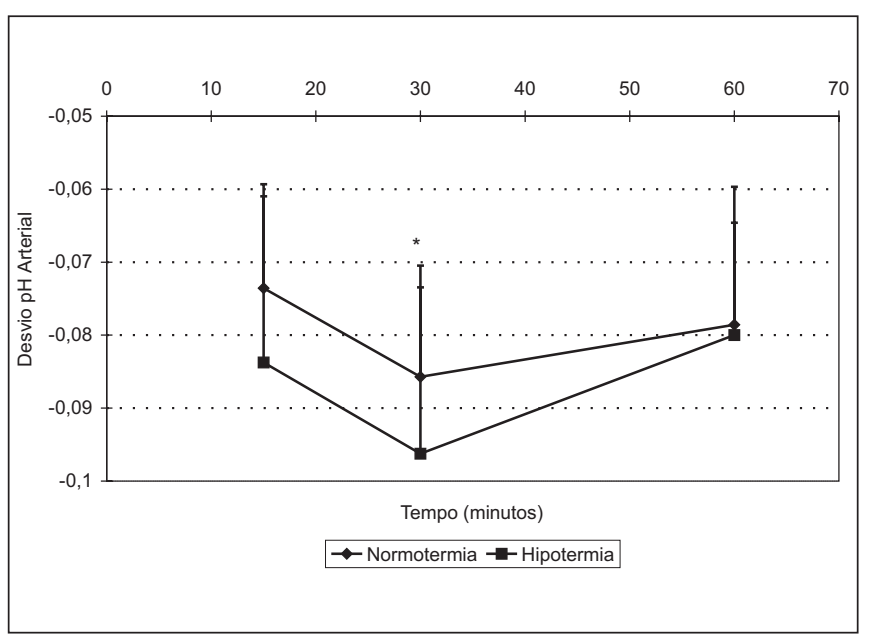

Figura 2 - Curvas dos Desvios Médios em Relação ao Tempo para o $\mathrm{pH}$ Arterial ${ }^{\star} p<0,05$

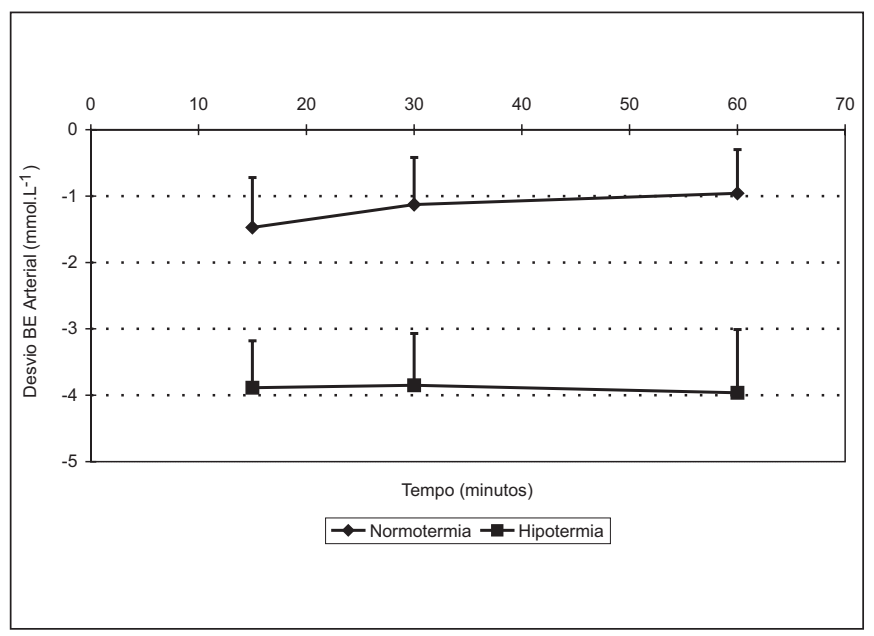

Figura 3 - Perfis Médios para o Excesso de Bases no Sangue Arterial

no intervalo entre 30 e 60 minutos, não foi detectada alteração estatisticamente significativa. Apesar disso, quando comparada ao tempo 15 minutos, a média geral no tempo 60 minutos foi a mesma pela análise estatística.

A variável "anion gap" (Figura 4) apresentou coincidência na evolução e na média dos desvios médios entre os grupos sem, no entanto, ter sido detectado efeito de tempo (médias dos tempos 15, 30 e 60 minutos não tiveram diferença estatisticamente significativa). O desvio médio geral ( \pm erro padrão) estimado ( $T$ ) é igual a -4,06 $\pm 0,76$.

Com relação à variável lactato, as medidas pré-CEC de cada grupo apresentam médias iguais $(p=0,5780)$, o mesmo não ocorrendo com as medidas pós-CEC $(p=0,0137)$. Os desvios médios de cada grupo parecem ser diferentes $(p=0,0159)$. Houve aumento significativo do lactato no grupo operado sob hipotermia.

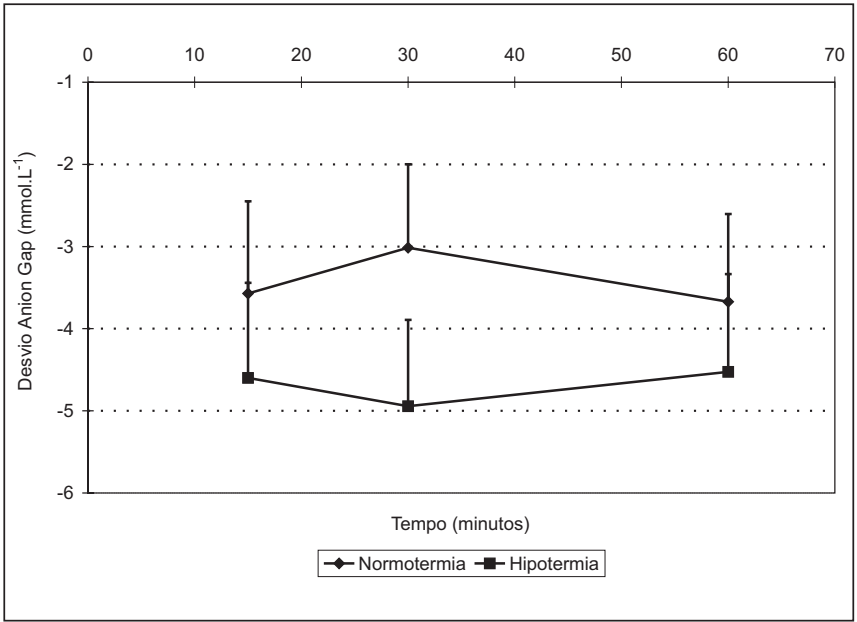

Figura 4 - Desvios Médios dos Valores do “Anion Gap” em Relação ao Tempo

\section{DISCUSSÃO}

Nesse estudo não foram encontradas diferenças estatisticamente significativas entre o grupo normotermia e o grupo hipotermia nos parâmetros $\mathrm{pH}$ arterial, bicarbonato plasmático, concentrações dos íons sódio e cloreto, e conteúdo arterial de oxigênio. Quanto à pressão parcial de $\mathrm{CO}_{2}$, parâmetro indicativo de possíveis alterações no $\mathrm{pH}$ por alterações ventilatórias, também não houve diferença estatisticamente significativa entre os dois grupos. As curvas para o excesso de bases e "anion gap" não apresentaram efeito de tempo, ao contrário das outras variáveis.

A despeito da igualdade entre os dois grupos para esses parâmetros, houve diferença estatisticamente significativa na concentração plasmática de potássio e no excesso de bases arteriais, ambos com valores médios menores no grupo hipotermia.

As técnicas de anestesia venosa total e balanceada mostram semelhança nas alterações do equilíbrio ácido-base e do íon lactato durante circulação extracorpórea com hipotermia moderada ${ }^{11}$. A circulação extracorpórea proporciona relativa manipulação do índice de fluxo arterial e da pressão arterial sistêmica através do fluxo da bomba de perfusão, além da infusão de fármacos vasoativos. Neste estudo, o fluxo foi mantido acima de $2 \mathrm{~L} \cdot \mathrm{min}^{-1} \cdot \mathrm{m}^{-2}$, durante a hipotermia, para proporcionar segurança adequada quanto a aumentos inesperados no consumo ou diminuição na liberação de oxigênio.

Em relação a concentração de lactato no sangue arterial, observou-se elevação dessa variável no período pós-CEC em relação ao controle pré-CEC, sugerindo que, apesar da hipotermia, o fluxo arterial foi inadequado. Também observou-se diferença significativa neste parâmetro entre os grupos no tempo pós-CEC, com valor médio mais elevado no grupo hipotermia, possivelmente indicando influência da temperatura. A explicação para esse efeito indesejável seria a elevação da resistência vascular sistêmica levando à diminuição da perfusão tecidual com concomitante aumento do metaboVol. 52, No 2, Março - Abril, 2002 
lismo anaeróbio e acúmulo de metabólitos ácidos ${ }^{12}$. Mesmo sem análise estatística, os valores obtidos de resistência vascular periférica nesse trabalho mostraram-se mais elevados durante a CEC no grupo hipotermia. A variável consumo de oxigênio, apesar de não submetida à análise estatística, mostrou valores em média mais elevados no grupo normotermia nos momentos estudados durante a CEC. Já foi relatado na literatura que, durante a CEC realizada pela técnica de hipotermia moderada, o consumo corpóreo de oxigênio diminui quando a temperatura corpórea é reduzida e que, além disso, a redução do fluxo a taxas de $1,2 \mathrm{~L} / \mathrm{min} / \mathrm{m}^{2} \mathrm{sob}$ condições moderadamente hipotérmicas não alteram o consumo de oxigênio ${ }^{13}$.

Não se observou diferença estatisticamente significativa entre os grupos normotermia e hipotermia em relação ao pH arterial, bicarbonato arterial, concentrações plasmáticas dos íons sódio e cloreto, "anion gap", pressão parcial de gás carbônico e conteúdo arterial de oxigênio. Houve efeito de tempo em todas essas variáveis, exceto para as variáveis excesso de bases e "anion gap".

O excesso de bases e a concentração de potássio variaram diferentemente nos grupos estudados, com o grupo hipotérmico apresentando valores inferiores aos do grupo normotérmico em todo período de circulação extracorpórea para ambas variáveis. O lactato sérico aumentou nos dois grupos do tempo Pré-CEC para o Pós-CEC e o grupo hipotermia apresentou valores mais elevados. Hipotermia leve, conforme utilizada neste trabalho, parece não modificar substancialmente o equilíbrio ácido-base, quando comparado a valores normais de temperatura, durante a circulação extracorpórea. Entretanto, o íon lactato, que reflete o metabolismo aeróbico celular, elevou-se significativamente nos pacientes operados sob hipotermia. Esta elevação pode sugerir que o transporte de oxigênio para a periferia não foi adequado durante período de observação proposto. Adiminuição da temperatura, embora discreta, parece não ter conferido o grau de proteção celular esperado ao fluxo sangüíneo calculado da circulação extracorpórea.

\section{Temperature and Acid-Base Balance in Coronary Bypass Grafting with Cardiopulmonary Bypass, under Hypothermia and Normothermia}

Hugo Leonardo de Moura Luz, M.D., José Otávio Costa Auler Junior, TSA, M.D.

\section{INTRODUCTION}

Cardiopulmonary bypass (CPB) is associated to several physiological changes. The multiple causes of these changes interact and are potential routes for postoperative organic dysfunction ${ }^{1}$.
During hypothermia, $\mathrm{pH}$ variations are biochemically independent of $\mathrm{PaCO}_{2}$ variations ${ }^{2}$.

Increased $\mathrm{pH}$ with decreased temperature, regardless of any changes in bicarbonate, hemoglobin or plasma protein changes, has been shown as early as in $1948^{3}$. In case of abnormal temperatures - and hypothermia is the best example $\mathrm{pH}$ changes may only mean cell and blood physiology adaptation ${ }^{4}$. The imidazol-histidin complex, in addition to determining blood neutrality with mild alkalinity over water's $\mathrm{pH}$, maintains, during temperature variations, an oscillation range, which is parallel and similar to water ${ }^{5}$. Physiological advantages of "alpha-stat" type control in men, who are homothermal animals, may be observed in experimental studies $^{6,7}$.

Cardiopulmonary bypass is always followed by a certain degree of myocardial dysfunction, even in the absence of hypothermia $^{1,8}$. Regardless of temperature or of the protocol to determine acid-base ( $\mathrm{pH}$-stat or alpha-stat) during CPB, $\mathrm{pH}$ of 7.4 and $\mathrm{PaCO}_{2}$ above $35 \mathrm{mmHg}$ is desirable at the end of rewarming for a safe pump disconnection. Acidosis may additionally depress myocardial contraction, decreasing inotropic action and increasing pulmonary vascular resistance.

Considering cardiopulmonary bypass physiological changes in cardiac surgery patients and possible changes in acid-base and metabolic balance, this study aimed at investigating changes in indicated parameters during cardiopulmonary bypass in hypothermia and at comparing them to those obtained in patients submitted to CPB under normothermia.

\section{METHODS}

After the Ethics Committee approval, this study was prospectively performed with patients submitted to myocardial revascularization under cardiopulmonary bypass in the Heart Institute, Hospital das Clinicas, Faculdade de Medicina, Universidade de São Paulo, from October 1999 to June 2000.

Participated in this study 30 adult patients of both genders, aged 41 to 78 years, scheduled for myocardial revascularization. Exclusion criteria were diabetes mellitus, renal failure or liver diseases.

Patients were distributed in two groups, according to the temperature used during cardiopulmonary bypass. Normothermia Group (N) - patients submitted to CPB in normothermia $\left(t=37{ }^{\circ} \mathrm{C}\right)$ and Hypothermia Group $(\mathrm{H})$ - patients submitted to CPB in hypothermia ( $t$ between 32 and 33 $\left.{ }^{\circ} \mathrm{C}\right)$. Patients' temperature was monitored with a nasopharyngeal sensor and continuously obtained during surgery. The study was performed in the intraoperative period and the following moments were evaluated:

M1 - Before cardiopulmonary bypass;

M2 -15 minutes after CPB;

M3 -30 minutes after CPB; 
M4 -60 minutes after CPB;

M5 - At the end of CPB (standardized in 15 minutes after protamine administration).

Arterial and venous blood samples were collected to measure hemoglobin $(\mathrm{Hb})$, blood gases concentration and acid-base balance; arterial and venous oxygen partial pressures $\left(\mathrm{PaO}_{2}\right.$ and $\left.\mathrm{PvO}_{2}\right)$, arterial and venous oxygen saturation $\left(\mathrm{SaO}_{2}\right.$ and $\left.\mathrm{SvO}_{2}\right)$, arterial and venous carbon dioxide partial pressures $\left(\mathrm{PaCO}_{2}\right.$ and $\left.\mathrm{PvCO}_{2}\right)$; arterial and venous $\mathrm{pH}$ ( $\mathrm{pHa}$ and $\mathrm{pHv}$ ), arterial and venous bicarbonate (Art bic and ven bic), and excess arterial and venous bases (BEa and BEv).

The anion gap was also calculated ( VN $=10$ to $\left.12 \mathrm{mmol}^{-1} \mathrm{~L}^{-1}\right)$ to check possible metabolic $\mathrm{pH}$ changes. Since the formula is $\mathrm{AG}=\mathrm{Na}^{+}-\left(\mathrm{Cl}^{-}+\mathrm{HCO}_{3}{ }^{+}\right)$, plasma sodium ion (flame photometry, $\mathrm{VN}=133$ to $145 \mathrm{mmol} . \mathrm{L}^{-1}$ ) and chloride (calorimetric method, $\mathrm{VN}=98$ to $119 \mathrm{mmol}$. $\mathrm{L}^{-1}$ ) were dosed.

Lactate ion was determined only for arterial blood in moments pre-CPB and post-CPB by the UV enzymatic method.

Tissue oxygenation and systemic vascular flow and resistance were calculated by adequate formulas for the three CPB periods:

\section{- oxygen content}

$\left(\mathrm{CaO}_{2}, \mathrm{VN}=17\right.$ to $20 \mathrm{ml} / \mathrm{dl}$ and $\mathrm{CvO}_{2}, \mathrm{VN}=12$ to 15 $\mathrm{ml} / \mathrm{dl}), \mathrm{CaO}_{2}=\left(\mathrm{Hb} \cdot \mathrm{SaO}_{2} \cdot 1.34 / 100\right)+\left(\mathrm{PaO}_{2} \cdot 0.0031\right) ;$

- arteriovenous oxygen difference $\left(\mathrm{DavO}_{2}, \mathrm{VN}=4\right.$ to 5 $\mathrm{ml} / \mathrm{dl}), \mathrm{DavO}_{2}=\mathrm{CaO}_{2}-\mathrm{CvO}_{2}$;

- oxygen extraction $\left(\mathrm{EO}_{2}, \mathrm{VN}=24\right.$ to $\left.28 \%\right), \mathrm{EO}_{2}=$ $\left(\mathrm{CaO}_{2}-\mathrm{CvO}_{2}\right) / \mathrm{CaO}_{2}$;

- oxygen transport index ( $\mathrm{iTO}_{2}, \mathrm{VN}=550$ to $\left.650 \mathrm{ml} / \mathrm{min} . \mathrm{m}^{2}\right)$, $\mathrm{iTO}_{2}=\left(\mathrm{CaO}_{2}\right.$ Flow $) /($ BodyArea.100);

- oxygen consumption index $\left(\mathrm{iVO}_{2}, \mathrm{VN}=115\right.$ a 165 $\left.\mathrm{ml} \cdot \mathrm{min}^{-1} \cdot \mathrm{m}^{2}\right), \mathrm{iVO}_{2}=\left(\operatorname{DavO}_{2} \cdot\right.$ Flow $) /($ BodyArea.100);

- flow index, iFlow=Flow/SupCorp;

- systemic vascular resistance index, iRVS = (MBP.80.1000)/(Flow/SupCorp).

Statistical analysis consisted of mean profile analysis of each group (normothermia and hypothermia) ${ }^{11}$. The variable studied was the deviation of the measurement compared to pre-CPB value, that is, response in time $x$ (minutes) is the difference between the value observed in time $x$ and pre-CPB value.

Post-CPB observations were not considered since they occurred in different times depending on the patient. The statistical model adopted has contemplated possible correlations between observations in the same patient; for such, a non-structured correlation was used ${ }^{9}$. Some interesting hypothesis were tested, such as, equal behavior between groups in moments studied (mean profiles coincidence test) ${ }^{10}$. Significance level was $5 \%$.

\section{RESULTS}

Table I shows demographic values for both normothermia ( $\mathrm{n}$ $=14)$ and hypothermia $(n=16)$ groups. No statistical differences were observed between groups.

Table I - Demographics Data (Mean \pm SD)

\begin{tabular}{lcc}
\hline & Group N & Group H \\
\hline Age (years) & $60.6 \pm 9.2$ & $62.5 \pm 10.3$ \\
Weight $(\mathrm{kg}$ & $77.6 \pm 6.8$ & $75.9 \pm 14.8$ \\
Height $(\mathrm{m})$ & $1.69 \pm 0.05$ & $1.68 \pm 0.06$ \\
Body surface $\left(\mathrm{m}^{2}\right)$ & $1.87 \pm 0.11$ & $1.86 \pm 0.21$ \\
\hline
\end{tabular}

$\mathrm{N}$ - Normothermia; H - Hypothermia

Mean CPB time and lowest temperature during $\mathrm{CPB}$ are shown in table II. CPB duration was longer for group $\mathrm{H}$.

Table II - Cardiopulmonary Bypass Parameters (Mean \pm SD)

\begin{tabular}{lcc}
\hline & Group N & Group H \\
\hline CPB duration (min) & $87 \pm 19$ & $105 \pm 25$ \\
Lowest temperature during CPB $\left({ }^{\circ} \mathrm{C}\right)$ & $35.2 \pm 1.2$ & $31.9 \pm 1.6$ \\
\hline
\end{tabular}

$\mathrm{N}$ - Normothermia; $\mathrm{H}$ - Hypothermia

Mean blood pressure, arterial flow and systemic vascular resistance were evaluated during the three predetermined CPB moments (15, 30 and 60 minutes) for both groups and mean values are shown in table III. Mean blood pressure and arterial flow were used to calculate other variables and were not statistically analyzed. Even without statistical analysis, peripheral vascular resistance was higher in group $\mathrm{H}$ during $\mathrm{CPB}$.

Table III - Physiological Parameters During CPB

CPB - 15 min CPB - 30 min CPB - 60 min

Mean blood pressure $(\mathrm{mmHg})$

\begin{tabular}{llll} 
Group N & $61.2 \pm 1.3$ & $56.6 \pm 1.3$ & $59.9 \pm 9.7$ \\
Group H & $61.2 \pm 9.3$ & $56.3 \pm 9.3$ & $60.0 \pm 9.7$ \\
\hline
\end{tabular}

Arterial flow during CPB (L. $\left.\min ^{-1} \cdot \mathrm{m}^{-2}\right)$

Group N $\quad 2575.5 \pm 523 \quad 2708.2 \pm 471 \quad 2648.9 \pm 556$

Group H $\quad 2497.8 \pm 350 \quad 2533.9 \pm 380 \quad 2414.6 \pm 332$

Systemic vascular resistance (dyn.seg. $\mathrm{cm}^{-5}$ )

Group N $\quad 2011.1 \pm 399 \quad 1868.8 \pm 586 \quad 2134.8 \pm 719$

\begin{tabular}{llll} 
Group H & $2128.4 \pm 378$ & $1957.9 \pm 454$ & $2169.7 \pm 533$ \\
\hline
\end{tabular}

Comparative data of $\mathrm{O}_{2}$ and $\mathrm{CO}_{2}$ partial pressures and tissue oxygenation parameters are shown in tables IV, $\mathrm{V}$ and $\mathrm{VI}$. Variables $\mathrm{PaO}_{2}, \mathrm{PvO}_{2}, \mathrm{PvCO}_{2}, \mathrm{CvO}_{2}, \mathrm{DavO}_{2}, \mathrm{iTO}_{2}$ and $\mathrm{EO}_{2}$ were not statistically analyzed and were only used to calculate other data.

Mean arterial partial $\mathrm{CO}_{2}$ pressures are shown in figure 1. Curves show parallelism and coincidence among them. The mean deviation increased in both groups in the interval between 15 and 30 CPB minutes without significant changes in the following period.

Revista Brasileira de Anestesiologia Vol. 52, № 2, Março - Abril, 2002 
TEMPERATURE AND ACID-BASE BALANCE IN CORONARY BYPASS GRAFTING WITH

CARDIOPULMONARY BYPASS, UNDER HYPOTHERMIA AND NORMOTHERMIA

Table IV - Oxygenation and Ventilation Parameters (Mean \pm SD)

\begin{tabular}{|c|c|c|c|c|c|}
\hline & Pre-CPB & CPB - 15 min & CPB - 30 min & CPB - $60 \mathrm{~min}$ & Post-CPB \\
\hline \multicolumn{6}{|c|}{ Arterial partial $\mathrm{O}_{2}$ pressure $(\mathrm{mmHg})$} \\
\hline Group N & $207.9 \pm 82.3$ & $200.3 \pm 72.4$ & $168.8 \pm 66.2$ & $187.3 \pm 60.4$ & $129.3 \pm 43.8$ \\
\hline Group H & $200.0 \pm 69.0$ & $179.7 \pm 78.9$ & $175.7 \pm 56.3$ & $179.0 \pm 64.5$ & $143.3 \pm 68.5$ \\
\hline \multicolumn{6}{|c|}{ Venous partial $\mathrm{O}_{2}$ pressure $(\mathrm{mmHg})$} \\
\hline Group N & $43.3 \pm 5.8$ & $40.9 \pm 6.4$ & $42.0 \pm 5.6$ & $40.8 \pm 5.3$ & $46.4 \pm 7.2$ \\
\hline Group H & $46.3 \pm 6.5$ & $45.9 \pm 6.6$ & $48.1 \pm 7.4$ & $43.8 \pm 8.6$ & $51.1 \pm 7.7$ \\
\hline \multicolumn{6}{|c|}{ Arterial partial $\mathrm{CO}_{2}$ pressure $(\mathrm{mmHg})$} \\
\hline Group N & $31.5 \pm 3.7$ & $37.9 \pm 3.3$ & $40.0 \pm 4.7^{*}$ & $39.6 \pm 5.1$ & $36.0 \pm 3.8$ \\
\hline Group H & $35.7 \pm 6.0$ & $39.9 \pm 5.6$ & $41.6 \pm 6.1^{*}$ & $38.8 \pm 6.0$ & $38.1 \pm 3.1$ \\
\hline \multicolumn{6}{|c|}{ Venous partial $\mathrm{CO}_{2}$ pressure $(\mathrm{mmHg})$} \\
\hline Group H & $42.4 \pm 5.1$ & $42.9 \pm 6.7$ & $44.7 \pm 7.7$ & $43.5 \pm 5.8$ & $42.5 \pm 4.2$ \\
\hline
\end{tabular}

${ }^{*} p<0.05$ for variation along time

Table V - Tissue Oxygenation Parameters Throughout the Study Period (Mean \pm SD)

\begin{tabular}{|c|c|c|c|c|c|}
\hline & Pre-CPB & CPB - 15 min & CPB - 30 min & CPB - 60 min & Post-CPB \\
\hline \multicolumn{6}{|c|}{ Arterial $\mathrm{O}_{2}$ content $(\mathrm{ml} / \mathrm{dl})$} \\
\hline Group N & $18.10 \pm 1.53$ & $13.14 \pm 2.61$ & $12.92 \pm 1.75$ & $13.58 \pm 1.69^{*}$ & $15.83 \pm 1.25$ \\
\hline Group H & $19.43 \pm 2.80$ & $12.75 \pm 1.28$ & $12.91 \pm 1.08$ & $13.48 \pm 1.40^{*}$ & $14.24 \pm 1.39$ \\
\hline \multicolumn{6}{|c|}{ Venous $\mathrm{O}_{2}$ content $(\mathrm{ml} / \mathrm{dl})$} \\
\hline Group N & $13.83 \pm 1.80$ & $9.86 \pm 2.17$ & $9.49 \pm 1.80$ & $9.76 \pm 1.91$ & $10.89 \pm 1.11$ \\
\hline Group H & $15.70 \pm 2.92$ & $9.80 \pm 1.61$ & $10.16 \pm 1.09$ & $10.05 \pm 1.57$ & $11.76 \pm 1.34$ \\
\hline \multicolumn{6}{|c|}{ Arteriovenous $\mathrm{O}_{2}$ difference $(\mathrm{ml} / \mathrm{dl})$} \\
\hline Group N & $4.27 \pm 1.18$ & $3.56 \pm 1.02$ & $3.43 \pm 0.88$ & $3.82 \pm 0.75$ & $2.93 \pm 0.92$ \\
\hline Group H & $3.73 \pm 1.02$ & $2.95 \pm 0.72$ & $2.75 \pm 0.64$ & $3.43 \pm 0.65$ & $2.48 \pm 0.76$ \\
\hline
\end{tabular}

${ }^{*} p<0.05$ for variation along time

Table VI - Tissue Oxygenation Parameters During CPB (Mean \pm SD)

\begin{tabular}{|c|c|c|c|}
\hline & CPB - 15 min & CPB - 30 min & CPB - 60 min \\
\hline \multicolumn{4}{|c|}{ Systemic vascular resistance $\left(\mathrm{din} / \mathrm{s} / \mathrm{cm}^{5}\right)$} \\
\hline Group N & $2011.1 \pm 399.0$ & $1868.8 \pm 585.7$ & $2134.8 \pm 718.6$ \\
\hline Group H & $2128.4 \pm 377.7$ & $1958.0 \pm 454.5$ & $2169.7 \pm 533.3$ \\
\hline \multicolumn{4}{|c|}{$\mathrm{O}_{2}$ transportation $\left(\mathrm{ml} / \mathrm{min} / \mathrm{m}^{2}\right)$} \\
\hline Group N & $323.32 \pm 107.95$ & $333.62 \pm 90.60$ & $344.18 \pm 103.32$ \\
\hline Group H & $297.43 \pm 48.51$ & $306.29 \pm 55.72$ & $303.60 \pm 48.39$ \\
\hline \multicolumn{4}{|c|}{$\mathrm{O}_{2}$ consumption $\left(\mathrm{ml} / \mathrm{min} / \mathrm{m}^{2}\right)$} \\
\hline Group N & $92.00 \pm 31.01$ & $91.92 \pm 26.28$ & $99.67 \pm 22.86$ \\
\hline Group H & $75.19 \pm 28.84$ & $69.04 \pm 17.36$ & $81.84 \pm 14.58$ \\
\hline \multicolumn{4}{|c|}{$\mathrm{O}_{2}$ extraction $(\%)$} \\
\hline Group H & $23.51 \pm 6.76$ & $21.31 \pm 4.94$ & $25.59 \pm 5.69$ \\
\hline
\end{tabular}




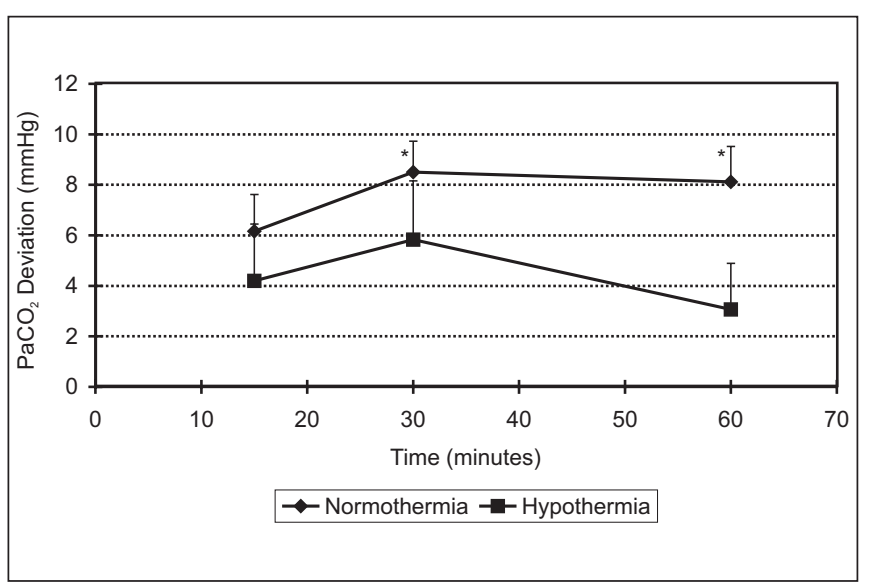

$\underset{*}{\text { Figure } 1}-\mathrm{Mean} \mathrm{PaCO}_{2}$ Profiles
( 0.05

$\mathrm{O}_{2}$ arterial content was similar for both groups and curves were coincident, showing no statistically significant differences between groups, with lower values for the hypothermia group. Between 30 and 60 minutes increase in arterial oxygen content was observed in both groups. Although not submitted to statistical analysis, oxygen consumption was, in average, higher in the normothermia group during CPB.
Blood $\mathrm{pH}$ and base excess were analyzed in arterial and venous blood and results are shown in table VII. Data on ion sodium, ion chloride and bicarbonate concentrations were obtained to calculate ion gap (AG). Lactate concentrations measured pre and post-CPB are also shown in table VII.

Arterial sodium ion concentration had a similar variation in both groups with statistically coincident curves. A significant increase in the mean deviation occurred in the interval between 30 and 60 minutes after CPB.

Potassium ion had a similar variation in serum concentration; however, mean values were statistically different between both groups with higher values during CPB for the normothermia group. An increase of the mean values was observed along time, showing a time-effect.

Chloride anion concentration has also shown parallel and coincident curves and temporal changes that were statistically significant only between 30 and 60 minutes after CPB.

Arterial $\mathrm{pH}$ had a similar variation between groups and mean profile curves along time (Figure 2) were coincident. Between 15 and 30 minutes of CPB a decrease of the mean values was observed although not statistically significant between 30 and 60 minutes, the last value being equal to that of 15 minutes.

Table VII - Acid-Base Balance Indicators (Mean \pm SD)

\begin{tabular}{|c|c|c|c|c|c|}
\hline & Pre-CPB & CPB -15 min & CPB - $30 \mathrm{~min}$ & CPB - $60 \mathrm{~min}$ & Post-CPB \\
\hline \multicolumn{6}{|c|}{ Arterial $\mathrm{pH}$} \\
\hline Group N & $7.45 \pm 0.068$ & $7.37 \pm 0.068$ & $7.36 \pm 0.077^{*}$ & $7.37 \pm 0.071$ & $7.35 \pm 0.077$ \\
\hline Group H & $7.46 \pm 0.039$ & $7.39 \pm 0.031$ & $7.37 \pm 0.042^{*}$ & $7.38 \pm 0.049$ & $7.38 \pm 0.035$ \\
\hline \multicolumn{6}{|c|}{ Arterial base excess (mmol.L- $\left.{ }^{1}\right)$} \\
\hline Group N & $-0.29 \pm 1.79$ & $-1.76 \pm 2.12$ & $-1.42 \pm 2.04$ & $-1.25 \pm 1.78$ & $-3.03 \pm 2.06^{* *}$ \\
\hline Group H & $1.85 \pm 3.01$ & $-2.04 \pm 2.07$ & $-2.00 \pm 2.50$ & $-2.11 \pm 2.92$ & $-3.97 \pm 3.83^{* *}$ \\
\hline \multicolumn{6}{|c|}{$\mathrm{Na}^{+}$concentration $\left(\mathrm{mmol} . \mathrm{L}^{-1}\right)$} \\
\hline Group N & $137.86 \pm 1.41$ & $129.36 \pm 3.20$ & $130.00 \pm 2.25$ & $131.00 \pm 2.18^{*}$ & $133.21 \pm 3.84$ \\
\hline Group H & $137.62 \pm 2.09$ & $130.81 \pm 3.21$ & $130.69 \pm 2.12$ & $131.75 \pm 1.73^{*}$ & $131.00 \pm 2.18$ \\
\hline \multicolumn{6}{|c|}{$\mathrm{K}^{+}$concentration $\left(\mathrm{mmol}^{-\mathrm{L}^{-1}}\right)$} \\
\hline Group N & $3.74 \pm 0.43$ & $4.28 \pm 0.39^{*}$ & $4.36 \pm 0.47^{*}$ & $4.66 \pm 0.48^{*}$ & $3.77 \pm 0.41^{* *}$ \\
\hline Group H & $3.90 \pm 0.30$ & $3.82 \pm 0.50^{*}$ & $4.16 \pm 0.67^{*}$ & $4.50 \pm 0.60^{*}$ & $3.76 \pm 0.36^{* *}$ \\
\hline \multicolumn{6}{|c|}{$\mathrm{Cl}^{-}$concentration $\left(\mathrm{mmol} \cdot \mathrm{L}^{-1}\right)$} \\
\hline Group N & $101.43 \pm 3.91$ & $97.93 \pm 3.07$ & $97.57 \pm 2.82$ & $98.93 \pm 2.30^{*}$ & $100.07 \pm 2.61$ \\
\hline Group H & $100.56 \pm 2.13$ & $98.87 \pm 2.75$ & $98.62 \pm 2.36$ & $99.50 \pm 2.78^{*}$ & $100.69 \pm 2.50$ \\
\hline \multicolumn{6}{|c|}{ Arterial $\mathrm{HCO}_{3}^{-}$concentration $\left(\mathrm{mmol}^{-1} \mathrm{~L}^{-1}\right)$} \\
\hline Group N & $23.00 \pm 1.75$ & $21.57 \pm 1.88$ & $22.01 \pm 2.01^{*}$ & $22.31 \pm 2.09$ & $19.94 \pm 2.09$ \\
\hline Group H & $23.30 \pm 2.16$ & $22.77 \pm 1.38$ & $23.24 \pm 1.54^{*}$ & $23.01 \pm 2.01$ & $21.44 \pm 2.85$ \\
\hline \multicolumn{6}{|c|}{ Anion Gap (mmol. $\left.\mathrm{L}^{-1}\right)$} \\
\hline Group N & $13.43 \pm 2.85$ & $9.86 \pm 3.05$ & $10.41 \pm 2.83$ & $9.76 \pm 3.07$ & $13.20 \pm 5.54$ \\
\hline Group H & $13.76 \pm 3.51$ & $9.16 \pm 3.06$ & $8.82 \pm 2.68$ & $9.24 \pm 2.85$ & $13.43 \pm 4.22$ \\
\hline \multicolumn{6}{|c|}{ Lactate ion $\left(\mathrm{mmol} . \mathrm{L}^{-1}\right)$} \\
\hline Group N & $15.27 \pm 6.04$ & & & & $25.43 \pm 11.16^{\star \star}$ \\
\hline Group H & $16.72 \pm 8.18$ & & & & $44.84 \pm 29.15^{\star *}$ \\
\hline
\end{tabular}

${ }^{*} p<0.05$ for variation along time

${ }^{* *} p<0.05$ for difference between groups 


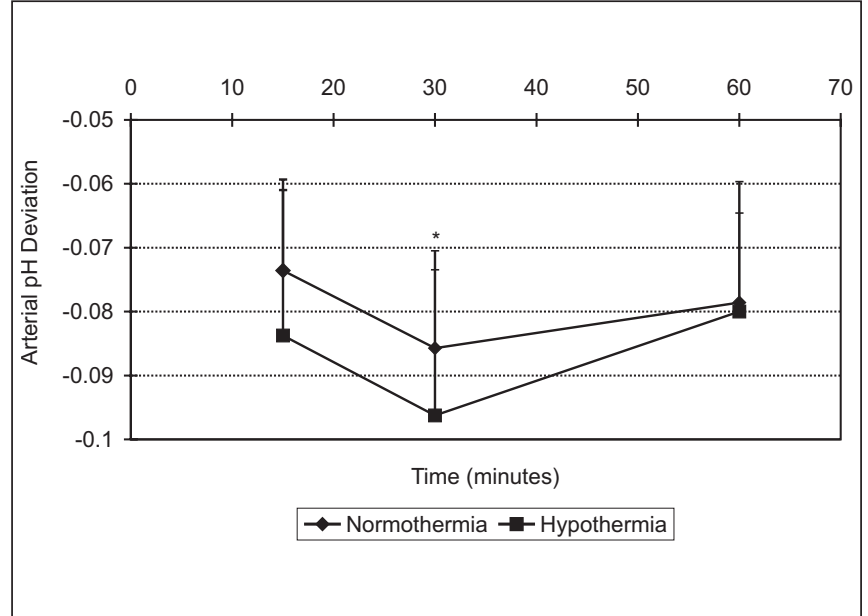

Figure 2 - Mean Deviation Curves Related to Time for Arterial $\mathrm{pH}$ ${ }^{*} p<0.05$

As to arterial bases excess, deviation curves for both groups were parallel and non-coincident and no changes along time have been detected (Figure 3).

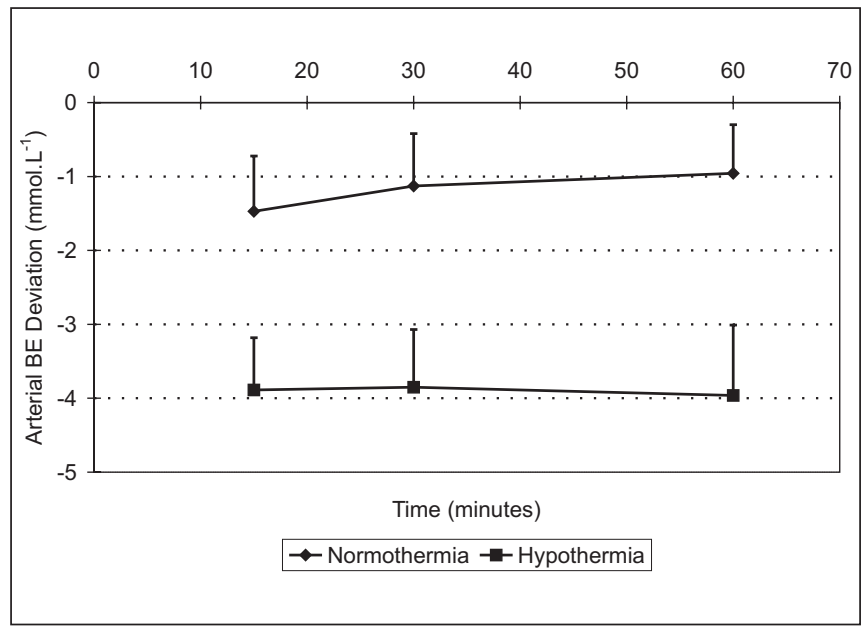

Figure 3 - Mean Arterial Base Excess Profiles

Arterial bicarbonate had similar variation along time for both groups. No statistically significant differences were observed between mean values of each curve, that is, they were coincident. Between 15 and 30 minutes after CPB an increase of the mean value was observed. Between 30 and 60 minutes no statistically significant changes were detected, however, when compared to 15 minutes, the 60 minutes mean value was statistically the same.

Anion gap (Figure 4) did not differ between groups (means at 15,30 and 60 minutes were not statistically different). The estimated mean deviation ( \pm standard deviation) ( $T$ ) was equal to $-4.06 \pm 0.76$.

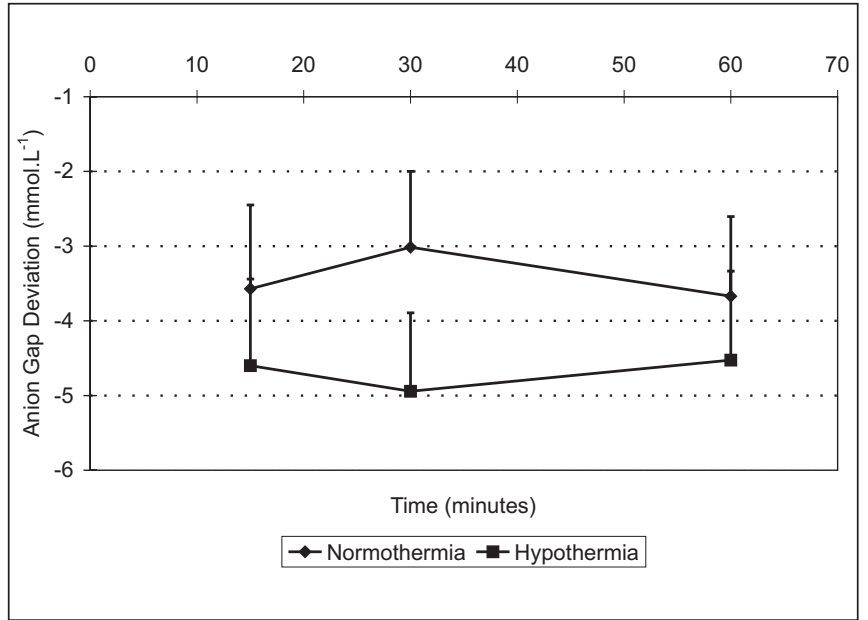

Figure 4 - Mean Anion Gap Deviations as Compared to Time

As to lactate, pre-CPB values did not differ between groups, the same being not true for post-CPB values ( $p=0.0137$ ). Mean deviations for each group are different $(p=0.0159)$. There has been a significant lactate increase in the hypothermia group.

\section{DISCUSSION}

Our study has not found statistically significant differences between groups in arterial $\mathrm{pH}$, plasma bicarbonate, sodium and chloride ion concentrations and oxygen arterial content. The $\mathrm{CO}_{2}$ partial pressure, which is an indicator of possible $\mathrm{pH}$ changes caused by ventilatory changes, did not show statistically significant differences between groups. Base excess and anion gap curves have not shown a time-effect, as opposed to other variables.

In spite of the equality between both groups for those parameters, a statistically significant lower mean value of potassium plasma concentration and arterial base excess was observed in the hypothermia group.

Total and balanced venous anesthesia techniques are similar in acid-base and lactate ion balance changes during cardiopulmonary bypass with moderate hypothermia ${ }^{11}$. CPB allows for a relative manipulation of arterial flow and systemic arterial pressure indices through the perfusion pump, in addition to the infusion of vasoactive drugs. In our study, the flow was maintained above $2 \mathrm{~L} \cdot \mathrm{min}^{-1} \cdot \mathrm{m}^{-2}$ during hypothermia to provide an adequate protection against unexpected increases in oxygen consumption or decreases in oxygen release.

Arterial lactate concentration was increased in the post-CPB period as compared to pre-CPB, suggesting that, in spite of hypothermia, arterial flow has been inadequate. There has also been a significant post-CPB difference in such parameter between groups, with a higher mean value in the hypothermia group, which could indicate the influence of temperature. The explanation for such undesirable effect would be an in- 
crease in systemic vascular resistance leading to a decreased tissue perfusion with a simultaneous increase in anaerobic metabolism and building up of acid metabolytes ${ }^{12}$. Even without statistical analysis, peripheral vascular resistance in this study was higher during CPB in the hypothermia group. Oxygen consumption, although not submitted to statistical analysis, showed higher mean values in the normothermia group during CPB. It has been reported in the literature that during CPB performed by the moderate hypothermia technique, body oxygen consumption is decreased when temperature is decreased and that, in addition, flow decrease to $1.2 \mathrm{~L}$. min. $\mathrm{m}^{2}$ under moderately hypothermal conditions does not change oxygen consumption ${ }^{13}$.

There were no statistically significant differences between groups of the arterial $\mathrm{pH}$, arterial bicarbonate, sodium and chloride ion plasma concentrations, anion gap, carbon dioxide partial pressure and arterial oxygen content. Except for base excess and anion gap, all other variables showed a time dependent effect.

Base excess and potassium concentration varied differently between groups, with lower values for the hypothermia group as compared to the normothermia group throughout cardiopulmonary bypass. Serum lactate increased in both groups at pre-CPB and post-CPB with higher values in the hypothermia group. Mild hypothermia, as used in this study, does not seem to substantially change acid-base balance, as compared to normal temperature values during cardiopulmonary bypass. Lactate ion, however, which reflects cell aerobic metabolism, was significantly higher in patients under hypothermia. This increase may suggest that oxygen transportation to periphery was not adequate during the proposed observation period. A decrease in temperature, although mild, does not seem to have provided adequate cell protection against calculated blood flow of cardiopulmonary bypass.

\section{REFERÊNCIAS - REFERENCES}

01. Moyers JR, Tinker JH - Emergence from Cardiopulmonary Bypass: Controversies about Physiology and Pharmacology, em: Tinker JH - Cardiopulmonary Bypass: Current Concepts and Controversies. A Society of Cardiovascular Anesthesiologists Monograph, WB Saunders, 1989:109-129.

02. Piccioni MA, Auler Jr JOC - Acid-base balance during hypothermia. B J Anesth Int Issue, 1993;4:56-62.

03. Rosenthal TB - The effects of temperature on the $\mathrm{pH}$ of blood and plasma in vitro. J Biol Chem, 1948;173:25-30.

04. Rahn H - Body temperature and acid-base regulation. Pneumologie, 1974; 151:87-94.

05. Reeves RB - An imidazole alphastat hypothesis for vertebrate acid-base regulation: tissue carbon dioxide content and body temperature in bullfrogs. Respir Physiol, 1972;14:219-236.

06. Polle-Wilson PA, Langer GA - Effect of $\mathrm{pH}$ on ionic exchange and function in rat and rabbit myocardium. Am J Physiol, 1975;229: 570-581.

07 . Sinet M, Muffat-Jolly M, Bendaace T et al - Maintaining blood pH at 7,4 during hypothermia has no significant effect on work of the isolated rat heart. Anesthesiology, 1985;62:582-587.
08. Hemmings HC, Thomas SJ - Termination of Cardiopulmonary Bypass, em: Gravlee GP, Davis RF, Utley JR - Cardiopulmonary Bypass. Principles and Practice. Baltimore, Williams \& Wilkins, 1993;760-784.

09. Singer JM, Andrade DF - Análise de dados longitudinais, em: VII Simpósio Nacional de Probabilidade e Estatística. São Paulo, Ed. UNICAMP, 1986;105.

10. Johnson RA, Wichern DW - Applied Multivariate Statistical Analysis. $3^{\text {rd }}$ Ed, New Jersey, Prentice Hall, 1992;642.

11. Moreira MRG - Estudo de Alterações Metabólicas durante a Circulação Extracorpórea com as técnicas de Anestesia Endovenosa Total e Balanceada. Dissertação de Mestrado apresentada à Faculdade de Medicina da Universidade de São Paulo, 1998.

12. Nordén I - The influence of anaesthetics on systemic vascular resistance during cardiopulmonary bypass. Scand J Thorac Cardiovasc Surg, 1974;8:81-87.

13. Hickey RF, Hoar PF - Whole-body oxygen consumption during low-flow hypothermic cardiopulmonary bypass. J Thorac Cardiovasc Surg, 1974;86:903-906.

\section{RESUMEN}

Luz HLM, Auler Jr JOC - Temperatura y Alteraciones en el Equilibrio Ácido-Base de Pacientes Sometidos a Cirugía Cardíaca con Circulación Extracorpórea, bajo Normotermia e Hipotermia

Justificativa y Objetivos - La circulación extracorpórea (CEC) se asocia a varios cambios en la fisiología normal. Las múltiplas causas de estas alteraciones interaccionan y representan un número de rutas potenciales para la disfunción orgánica pós-operatoria. El objetivo de este trabajo es investigar las alteraciones de sus parámetros indicadores durante a circulación extracorpórea en hipotermia y compararlas a aquellas ocurridas en normotermia.

Método - Fueron seleccionados 30 pacientes adultos, de ambos sexos, con edades entre 41 y 78 años, indicados para la revascularización quirúrgica del miocárdio, operados con auxilio de CEC, bajo normotermia o hipotermia. Fueron evaluados los siguientes parámetros: concentración de hemoglobina y de los gases sanguíneos, $\mathrm{pH}$, bicarbonato, exceso de bases, hiato aniónico, íon lactato, parámetros de oxigenación tecidual y los índices de flujo y de resistencia vascular sistémica.

Resultados - No hubo diferencia estadísticamente significativa entre los grupos normotermia e hipotermia, en relación al $\mathrm{pH}$ arterial, al bicarbonato arterial, a las concentraciones plasmáticas de los íons sodio y cloreto, al "anion gap", a la presión parcial de gás carbónico y al contenido arterial de oxígeno. Hubo efecto de tiempo en todas esas variables excepto para las variables exceso de bases y "anion gap". El exceso de bases y la concentración de potasio mostraran valores inferiores en el grupo hipotérmico. El lactato sérico aumentó en los dos grupos del tiempo antes para el después la CEC siendo que el grupo hipotermia presentó valores más elevados.

Conclusiones - Hipotermia leve parece no modificar substancialmente el equilibrio ácido-base, cuando comparado a valores normales de temperatura, durante a CEC. Entretanto, el íon lactato se elevó significativamente en los pacientes operados sobre hipotermia, sugiriendo que el transporte de oxígeno para la periferia no fue adecuado durante el período de observación propuesto. La disminución de la temperatura, aun cuando discreta, parece no haber conferido el grado de protección celular esperada al flujo sanguíneo de la CEC. 